\title{
Electrodynamic Effects of Inflationary Gravitons
}

\author{
D. Glavan* and T. Prokoped \\ Institute for Theoretical Physics $\&$ Spinoza Institute \\ Utrecht University, Postbus 80195, 3508 TD Utrecht, THE NETHERLANDS \\ S. P. Miad $\ddagger$ \\ Department of Physics, National Cheng Kung University \\ No.1, University Road, Tainan City 701, TAIWAN \\ R. P. Woodard $\S$ \\ Department of Physics, University of Florida, \\ Gainesville, 32611 FL, UNITED STATES
}

\begin{abstract}
We calculate the one-loop corrections from inflationary gravitons to the electromagnetic fields of a point charge and a point magnetic dipole on a locally de Sitter space background. Results are obtained both for an observer at rest in co-moving coordinates, whose physical distance from the sources increases with the expanding universe, and for an observer at rest in static coordinates, whose physical distance from the sources is constant. The fields of both sources show the de Sitter analogs of the fractional $G / r^{2}$ corrections which occur in flat space, but there are also some fractional $G H^{2}$ corrections due to the scattering of virtual photons from the vast ensemble of infrared gravitons produced by inflation. The co-moving observer perceives the magnitude of the point charge to increase linearly with co-moving time and logarithmically with the co-moving position, however, the magnetic dipole shows only a negative logarithmic spatial variation. The static observer perceives no secular change of the point charge but he does report a secular enhancement of the magnetic dipole moment.
\end{abstract}

\footnotetext{
* d.glavan@uu.nl

$\dagger$ t.prokopec@uu.nl

$\ddagger$ spmiao5@mail.ncku.edu.tw

§ woodard@phys.ufl.edu
} 


\section{Introduction}

Primordial inflation produces a vast ensemble of scalars and gravitons which are the sources of primordial scalar and tensor perturbations [1]. These ensembles can alter the properties of particles and the forces they carry. Many studies of these modifications have been made in recent years, both for scalar-mediated effects [2]6] and for graviton-mediated effects [7-11]. The aim of this paper is to determine the leading corrections from inflationary gravitons to the electric and magnetic fields produced by a point charge and by a point magnetic dipole. Our technique is to solve the quantum-corrected Maxwell's equation,

$$
\partial_{\nu}\left[\sqrt{-g} g^{\nu \alpha} g^{\mu \beta} F_{\alpha \beta}(x)\right]+\int d^{4} x^{\prime}\left[{ }^{\mu} \Pi_{R}^{\nu}\right]\left(x ; x^{\prime}\right) A_{\nu}\left(x^{\prime}\right)=J^{\mu}(x)
$$

where $F_{\mu \nu}=\partial_{\mu} A_{\nu}-\partial_{\nu} A_{\mu}$ is the electromagnetic field strength tensor, $A_{\mu}$ is the electromagnetic 4-potential, $J^{\mu}$ is the source 4-current, $i\left[{ }^{\mu} \Pi_{R}^{\nu}\right]\left(x ; x^{\prime}\right)$ is the retarded vacuum polarization induced by the interactions with gravitons. We infer the retarded vacuum polarization using the Schwinger-Keldysh formalism [12] from a recent computation of the one loop graviton contribution to the vacuum polarization on de Sitter background, made using dimensional regularization and BPHZ (Bogoliubov-Parasiuk-Hepp-Zimmermann) renormalization [13]. The relevant diagrams are displayed in Fig. 1,

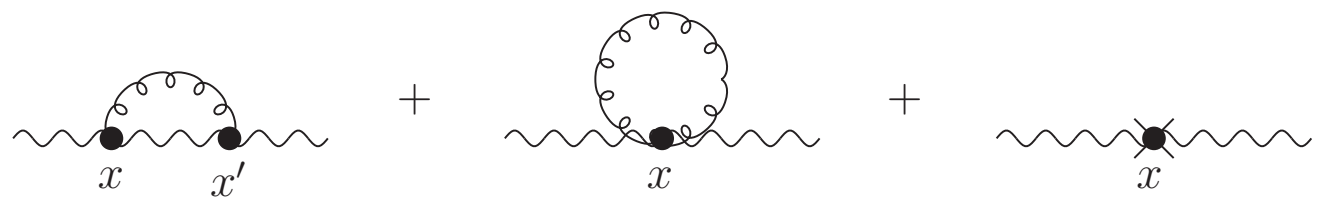

FIG. 1. Feynman diagrams relevant to the one loop vacuum polarization from gravitons. Wavy lines are photons and curly lines are gravitons.

Our analysis is highly relevant to three earlier works [6, 10, 14]. The last of these is a study of graviton corrections to electromagnetism on flat space background. Because the same sources were included, this work gives the flat space correspondence limits of our de Sitter results. Static fields in flat space can only depend upon the distance $r$ from the source, so one loop quantum gravitational corrections must be proportional to the classical result times $G / r^{2}$, where $G$ is Newton's constant. Of course explicit computation [14, 15] confirms this simple consequence of dimensional analysis.

Our de Sitter problem has another dimensional parameter in the form of the Hubble constant $H$. It can also show secular growth, deriving ultimately from the fact that more and more gravitons are ripped out of the vacuum as inflation progresses. These features mean the classical fields can suffer fractional corrections of the form $G H^{2} \times \ln (a)$, where $a(t)=e^{H t}$ is the de Sitter scale factor. Because those corrections have the same spatial dependence as the classical result it seems fair to regard them as time dependent renormalizations 
of the classical sources, which in our case are the charge and the magnetic dipole moment. Precisely this sort of secular renormalization was seen in a study of the effect of charged inflationary scalars on the same two sources [6]. Because that study found different results for observers at a fixed physical distance from the source and those who are being pulled away by the inflationary expansion, we shall also derive results for both cases. And a major motivation for our work is to check the recent claim by Kitamoto and Kitazawa that inflationary gravitons screen gauge coupling constants [10].

Section II of this paper recasts the results of Ref. [13] in Schwinger-Keldysh form [12] to give the one loop retarded vacuum polarization. In section III we make a loop expansion on the field strength of the effective field and derive an integral expression for the one loop contribution in terms of the tree order field strengths and the structure functions of the vacuum polarization. Section IV gives the actual derivation of the field strengths for a point charge and for a point magnetic dipole, with some technical details consigned to appendices. Our conclusions comprise section V.

\section{The Retarded Vacuum Polarization}

The de Sitter metric tensor in spatially flat, conformal coordinates is $g_{\mu \nu}=a^{2}(\eta) \eta_{\mu \nu}$. Here and henceforth, the Minkowski metric is $\eta_{\mu \nu}=\operatorname{diag}(-1,1,1,1)$, and $a(\eta)=-1 /(H \eta)$ is the scale factor in terms of conformal time $\eta$. Because the vacuum polarization is a transverse bi-vector density it can be written in the form [3],

$$
i\left[{ }^{\mu} \Pi^{\nu}\right]\left(x ; x^{\prime}\right)=\left(\eta^{\mu \nu} \eta^{\rho \sigma}-\eta^{\mu \sigma} \eta^{\nu \rho}\right) \partial_{\rho} \partial_{\sigma^{\prime}} F\left(x ; x^{\prime}\right)+\left(\bar{\eta}^{\mu \nu} \bar{\eta}^{\rho \sigma}-\bar{\eta}^{\mu \sigma} \bar{\eta}^{\nu \rho}\right) \partial_{\rho} \partial_{\sigma^{\prime}} G\left(x ; x^{\prime}\right)
$$

Here and henceforth, placing a bar over a tensor indicates that its temporal components have been suppressed, for example, $\bar{\eta}^{\mu \nu} \equiv \eta^{\mu \nu}+\delta_{0}^{\mu} \delta_{0}^{\nu} . F\left(x ; x^{\prime}\right)$ and $G\left(x ; x^{\prime}\right)$ are known as structure functions, and one can show that two of them are needed if the only coordinate symmetries are homogeneity and isotropy [16]. Because the graviton propagator breaks de Sitter invariance [17, 18], a de Sitter breaking representation like (21) is mandatory. Had all ten of the de Sitter isometries been present one could employ a hugely more complicated but de Sitter invariant representation involving only a single structure function. However, this representation seems to obscure, rather than elucidate, the essential physics [16]. And a simple procedure exists for transforming between different representations [19].

The one loop graviton contributions to the structure functions of the renormalized, in-out vacuum polarization were given in Eqs. (136-137) of Ref. [13]. After some straightforward rearrangements, those results 
can be expressed as,

$$
\begin{gathered}
F\left(x ; x^{\prime}\right)=\frac{\kappa^{2}}{8 \pi^{2}}\left\{H^{2}[\ln (a)+\alpha]+\frac{1}{a^{2}}\left[-\frac{1}{3} \ln (a)+\beta\right]\left[\partial^{2}+2 H a \partial_{0}\right]+\frac{H}{3 a} \partial_{0}\right\} i \delta^{4}\left(x-x^{\prime}\right) \\
-\frac{\kappa^{2}}{1536 \pi^{4}} \frac{1}{a} \partial^{6}\left\{\frac{1}{a^{\prime}}\left[\ln ^{2}\left(\frac{H^{2}}{4} \Delta x^{2}\right)-2 \ln \left(\frac{H^{2}}{4} \Delta x^{2}\right)\right]\right\} \\
+\frac{\kappa^{2} H^{2}}{128 \pi^{4}}\left\{\left[\frac{1}{4} \partial^{4}+\partial^{2} \partial_{0}^{2}\right] \ln ^{2}\left(\frac{1}{4} H^{2} \Delta x^{2}\right)+\left[-\frac{1}{2} \partial^{4}+2 \partial^{2} \partial_{0}^{2}\right] \ln \left(\frac{1}{4} H^{2} \Delta x^{2}\right)\right\}+O\left(\kappa^{4}\right), \\
G\left(x ; x^{\prime}\right)=\frac{\kappa^{2} H^{2}}{6 \pi^{2}}\left[-\ln (a)+\frac{3}{4} \gamma\right] i \delta^{4}\left(x-x^{\prime}\right) \\
-\frac{\kappa^{2} H^{2}}{384 \pi^{4}} \partial^{4}\left\{\ln ^{2}\left(\frac{1}{4} H^{2} \Delta x^{2}\right)-2 \ln \left(\frac{1}{4} H^{2} \Delta x^{2}\right)\right\}+O\left(\kappa^{4}\right) .
\end{gathered}
$$

Here the loop counting parameter of quantum gravity is $\kappa^{2}=16 \pi G$, the flat space d'Alembertian is $\partial^{2} \equiv$ $\eta^{\mu \nu} \partial_{\mu} \partial_{\nu}$ and we define the invariant interval $\Delta x^{2}\left(x ; x^{\prime}\right)$ as,

$$
\Delta x^{2}=-\left(\left|\eta-\eta^{\prime}\right|-i \epsilon\right)^{2}+\left\|\vec{x}-\vec{x}^{\prime}\right\|^{2}
$$

The parameters $\alpha, \beta$ and $\gamma$ in (3,4) are finite renormalization constants that are related to the one loop counterterms used in Ref. [13],

$$
\begin{aligned}
\mathcal{L}_{\mathrm{ct}}= & C_{1} \sqrt{-g} R F_{\mu \nu} F^{\mu \nu}+C_{2} \sqrt{-g} R^{\nu \sigma} F_{\mu \nu} F_{\rho \sigma} g^{\mu \rho}+C_{3} \sqrt{-g} R^{\mu \nu \rho \sigma} F_{\mu \nu} F_{\rho \sigma} \\
& +C_{4} \sqrt{-g}\left(\nabla_{\alpha} F_{\mu \nu}\right)\left(\nabla^{\alpha} F^{\mu \nu}\right)+\Delta C H^{2} \sqrt{-g} F_{i j} F_{k l} g^{i k} g^{j l} .
\end{aligned}
$$

In de Sitter background only the combination $\bar{C}=D(D-1) C_{1}+(D-1) C_{2}+2 C_{3}$ matters. We define $C_{4 f}, \bar{C}_{f}$ and $\Delta C_{f}$ as the finite parts of each coefficient, and the parameters $\alpha, \beta$ and $\gamma$ are,

$$
\begin{array}{r}
\alpha=\frac{1}{2}\left[3 \ln \left(\frac{4 \mu^{2}}{H^{2}}\right)-4\right]+\frac{32 \pi^{2}}{\kappa^{2}}\left(\bar{C}_{f}-4 C_{4 f}\right) \quad, \quad \beta=-\frac{1}{6} \ln \left(\frac{4 \mu^{2}}{H^{2}}\right)-\frac{32 \pi^{2}}{\kappa^{2}} C_{4 f}, \\
\gamma=\frac{1}{3}\left[\ln \left(\frac{4 \mu^{2}}{H^{2}}\right)-\frac{33}{2}\right]+\frac{32 \pi^{2}}{\kappa^{2}}\left(\Delta C_{f}-2 C_{4 f}\right) .
\end{array}
$$

Had Einstein + Maxwell been a renormalizable theory we could have invoked some physical renormalization condition to fix the coefficients $\alpha, \beta$ and $\gamma$. However, Einstein + Maxwell is not perturbatively renormalizable [20], so we must instead treat it in the sense of low energy effective field theory [21]. That is, we regard the finite renormalization constants $\alpha, \beta$ and $\gamma$ as arbitrary free parameters which characterize our ignorance of the true ultraviolet completion of Einstein + Maxwell, and we trust only predictions of the theory which are insensitive to the values of these parameters. Precisely this was done by Bjerrum-Bohr in his computation of the long range one loop graviton contribution to the Coulomb potential on a flat background [15]. We will comment further on this at the appropriate points of subsections IV A and IV B, after our full results for the one loop field strengths have been derived and it is possible to identify regimes in which the unambiguous contributions dominate those from $\alpha, \beta$ and $\gamma$. 
Using the in-out structure functions (3,4) in Eq. (11) would be appropriate for a flat space scattering problem but it makes little sense in cosmology where the universe began with an initial singularity and no one knows its final state. Using the in-out structure functions would make the effective field equations depend strongly on the far future; it would also result in the electromagnetic field strengths developing imaginary parts. The more appropriate problem to study in cosmology is what happens to the field strengths when the universe is released in a prepared state at some finite time. The appropriate structure functions for this sort of problem are the retarded ones of the Schwinger-Keldysh formalsim [12]. [22],

Fortunately, there is a very simple procedure for converting in-out structure functions into retarded ones

$$
F_{R}\left(x ; x^{\prime}\right)=F_{++}\left(x ; x^{\prime}\right)+F_{+-}\left(x ; x^{\prime}\right), \quad G_{R}\left(x ; x^{\prime}\right)=G_{++}\left(x ; x^{\prime}\right)+G_{+-}\left(x ; x^{\prime}\right)
$$

We extract the ++ and +- structure functions from (3) 4 by replacing the invariant interval $\Delta x^{2}\left(x ; x^{\prime}\right)$ with,

$$
\Delta x_{++}^{2}\left(x ; x^{\prime}\right) \equiv-\left(\left|\eta-\eta^{\prime}\right|-i \epsilon\right)^{2}+\left\|\vec{x}-\vec{x}^{\prime}\right\|^{2} \quad, \quad \Delta x_{+-}^{2}\left(x ; x^{\prime}\right) \equiv-\left(\eta-\eta^{\prime}+i \epsilon\right)^{2}+\left\|\vec{x}-\vec{x}^{\prime}\right\|^{2} .
$$

We also drop the delta function terms in the +- case, and introduce an overall minus sign. The result is,

$$
\begin{aligned}
-i F_{R}\left(x ; x^{\prime}\right)= & \frac{\kappa^{2}}{8 \pi^{2}}\left\{H^{2}[\ln (a)+\alpha]+\frac{1}{a^{2}}\left[-\frac{1}{3} \ln (a)+\beta\right]\left[\partial^{2}+2 H a \partial_{0}\right]+\frac{H}{3 a} \partial_{0}\right\} \delta^{4}\left(x-x^{\prime}\right) \\
& -\frac{\kappa^{2}}{384 \pi^{3}} \frac{1}{a} \partial^{6}\left\{\frac{1}{a^{\prime}} \theta\left(\Delta \eta-\left\|\vec{x}-\vec{x}^{\prime}\right\|^{2}\right)\left[\ln \left[\frac{H^{2}}{4}\left(\Delta \eta^{2}-\left\|\vec{x}-\vec{x}^{\prime}\right\|^{2}\right)\right]-1\right]\right\} \\
& +\frac{\kappa^{2} H^{2}}{32 \pi^{3}}\left\{\left[\frac{1}{4} \partial^{4}+\partial^{2} \partial_{0}^{2}\right] \theta\left(\Delta \eta-\left\|\vec{x}-\vec{x}^{\prime}\right\|\right) \ln \left[\frac{H^{2}}{4}\left(\Delta \eta^{2}-\left\|\vec{x}-\vec{x}^{\prime}\right\|^{2}\right)\right]\right. \\
& \left.+\left[-\frac{1}{4} \partial^{4}+\partial^{2} \partial_{0}^{2}\right] \theta\left(\Delta \eta-\left\|\vec{x}-\vec{x}^{\prime}\right\|\right)\right\}+O\left(\kappa^{4}\right), \\
-i G_{R}\left(x ; x^{\prime}\right)= & \frac{\kappa^{2} H^{2}}{8 \pi^{2}}\left[-\frac{4}{3} \ln (a)+\gamma\right] \delta^{4}\left(x-x^{\prime}\right) \\
& -\frac{\kappa^{2} H^{2}}{96 \pi^{3}} \partial^{4}\left\{\theta\left(\Delta \eta-\left\|\vec{x}-\vec{x}^{\prime}\right\|\right)\left[\ln \left[\frac{H^{2}}{4}\left(\Delta \eta^{2}-\left\|\vec{x}-\vec{x}^{\prime}\right\|^{2}\right)\right]-1\right]\right\}+O\left(\kappa^{4}\right) .
\end{aligned}
$$

To reach these forms we have used the identities,

$$
\begin{aligned}
& \ln \left(\frac{H^{2}}{4} \Delta x_{++}^{2}\right)-\ln \left(\frac{H^{2}}{4} \Delta x_{+-}^{2}\right)=2 i \pi \theta\left(\Delta \eta-\left\|\vec{x}-\vec{x}^{\prime}\right\|\right), \\
& \ln ^{2}\left(\frac{H^{2}}{4} \Delta x_{++}^{2}\right)-\ln ^{2}\left(\frac{H^{2}}{4} \Delta x_{+-}^{2}\right)=4 i \pi \theta\left(\Delta \eta-\left\|\vec{x}-\vec{x}^{\prime}\right\|\right) \ln \left[\frac{H^{2}}{4}\left[\Delta \eta^{2}-\left\|\vec{x}-\vec{x}^{\prime}\right\|^{2}\right]\right] .
\end{aligned}
$$

The retarded structure functions $-i F_{R}\left(x ; x^{\prime}\right)$ and $-i G_{R}\left(x ; x^{\prime}\right)$ in (10) 11) and the corresponding vacuum polarization tensor are manifestly real and causal (in the sense that they vanish outside the past light-cone).

These rules correspond to releasing the universe in free vacuum. If the initial state has corrections — as it must when interactions are present - there will be interactions on the initial value surface [23]. With the simple representation (2) we are using, these temporal surface terms should fall off like powers of 1/a [16]. 
Because we are only interested in the asymptotic late time forms of the quantum-corrected field strengths we will not bother correcting the initial state.

\section{One Loop Effective Field Equation}

We can rewrite the effective field equation (1) in a convenient form by noting that all the scale factors cancel in $\sqrt{-g} g^{\nu \alpha} g^{\mu \beta}=\eta^{\nu \alpha} \eta^{\mu \beta}$, by plugging in the vacuum polarization (2), and by partially integrating the primed derivatives,

$$
\partial_{\nu} F^{\nu \mu}(x)=J^{\mu}(x)-\partial_{\nu} \int d^{4} x^{\prime}\left[i F_{R}\left(x ; x^{\prime}\right) F^{\nu \mu}\left(x^{\prime}\right)+i G_{R}\left(x ; x^{\prime}\right) \bar{F}^{\nu \mu}\right] .
$$

Here and henceforth the indices on the field strength tensor are raised with the Minkowski metric, $F^{\mu \nu}=$ $\eta^{\mu \alpha} \eta^{\nu \beta} F_{\alpha \beta}$, and we remind that reader that an overline indicates the tensor has its temporal components suppressed, $\bar{F}^{\mu \nu} \equiv \bar{\eta}^{\mu \alpha} \bar{\eta}^{\nu \beta} F_{\alpha \beta}$, with $\bar{\eta}^{\mu \nu} \equiv \eta^{\mu \nu}+\delta_{0}^{\mu} \delta_{0}^{\nu}$.

Because we only know the structure functions at order $\kappa^{2}$ there is no alternative to solving (14) in the loop expansion,

$$
\begin{aligned}
F^{\mu \nu}(x) & =F_{(0)}^{\mu \nu}(x)+\kappa^{2} F_{(1)}^{\mu \nu}(x)+\mathcal{O}\left(\kappa^{4}\right), \\
F_{R}\left(x ; x^{\prime}\right) & =0+\kappa^{2} F_{(1)}\left(x ; x^{\prime}\right)+\mathcal{O}\left(\kappa^{4}\right) \\
G_{R}\left(x ; x^{\prime}\right) & =0+\kappa^{2} G_{(1)}\left(x ; x^{\prime}\right)+\mathcal{O}\left(\kappa^{4}\right) .
\end{aligned}
$$

We assume the current density $J^{\mu}(x)$ is classical, so the zeroth order and the first order equations are,

$$
\begin{aligned}
& \partial_{\nu} F_{(0)}^{\nu \mu}(x)=J^{\mu}(x) \\
& \partial_{\nu} F_{(1)}^{\nu \mu}(x)=\partial_{\nu} \int d^{4} x^{\prime}\left[-i F_{(1)}\left(x ; x^{\prime}\right) F_{(0)}^{\nu \mu}\left(x^{\prime}\right)-i G_{(1)}\left(x ; x^{\prime}\right) \bar{F}_{(0)}^{\nu \mu}\left(x^{\prime}\right)\right] .
\end{aligned}
$$

Given the source $J^{\mu}(x)$ one solves equation (18) to find the classical field strengths $F_{(0)}^{\nu \mu}(x)$, then one uses this in (19), together with the order $\kappa^{2}$ parts of (10,11), to solve for the one loop field strengths.

An important intermediate step is working out the primed integral on the right hand side of (19)), without the unprimed derivative,

$$
\mathcal{F}^{\mu \nu}(x) \equiv \int d^{4} x^{\prime}\left[-i F_{(1)}\left(x ; x^{\prime}\right) F_{(0)}^{\mu \nu}\left(x^{\prime}\right)-i G_{(1)}\left(x ; x^{\prime}\right) \bar{F}_{(0)}^{\mu \nu}\left(x^{\prime}\right)\right]
$$

where we assume the initial time is $\eta_{0}=-1 / H$. (The Heaviside theta functions in Eqs. (10 13) then dictate that the integration over $\eta^{\prime}$ is from $-1 / H$ to $\eta$.) The divergence of $\mathcal{F}^{\mu \nu}$ defines what we might call the one loop current $J_{(1)}^{\mu}$, which sources the one loop field strength,

$$
J_{(1)}^{\mu}(x) \equiv \partial_{\rho} \mathcal{F}^{\rho \mu}(x) \quad \Longrightarrow \quad \partial^{2} F_{(1)}^{\mu \nu}(x)=\partial^{\mu} J_{(1)}^{\nu}(x)-\partial^{\nu} J_{(1)}^{\mu}(x)
$$


Because the retarded Green's function of $\partial^{2},-\delta\left(\eta-\eta^{\prime}-\left\|\vec{x}-\vec{x}^{\prime}\right\|\right) / 4 \pi\left\|\vec{x}-\vec{x}^{\prime}\right\|$, is translation invariant in space and time, we can partially integrate the primed derivatives 1 and then reflect them to unprimed derivatives to reach the form,

$$
F_{(1)}^{\mu \nu}=\partial^{\mu} \partial_{\rho} \frac{1}{\partial^{2}} \mathcal{F}^{\rho \nu}-\partial^{\nu} \partial_{\rho} \frac{1}{\partial^{2}} \mathcal{F}^{\rho \mu}
$$

An important simplification occurs when the classical field strengths derive from static scalar or vector potentials. In that case one can partially integrate the spatial derivatives onto the one loop structure functions $F_{(1)}\left(x ; x^{\prime}\right)$ and $G_{(1)}\left(x ; x^{\prime}\right)$, and then exploit homogeneity to reflect them into unprimed derivatives. For the case of a static scalar potential, with zero vector potential, we find,

$$
A_{(0)}^{\mu}(\eta, \vec{x})=\left(-\Phi_{(0)}(\vec{x}), \overrightarrow{0}\right) \Longrightarrow \mathcal{F}^{0 i}=-\partial_{i} \int d^{4} x^{\prime} i F_{(1)}\left(x ; x^{\prime}\right) \Phi_{(0)}\left(\vec{x}^{\prime}\right) \equiv-\partial_{i} \mathcal{J}_{\Phi} \quad, \quad \mathcal{F}^{i j}=0
$$

Substituting (23) into (22) tells us that the one loop field strengths agree with $\mathcal{F}^{\mu \nu}$,

$$
\begin{aligned}
& A_{(0)}^{\mu}(\eta, \vec{x})=\left(-\Phi_{(0)}(\vec{x}), \overrightarrow{0}\right) \\
& \quad \Longrightarrow F_{(1)}^{0 i}=\partial^{0} \partial_{0} \frac{1}{\partial^{2}} \mathcal{F}^{0 i}-\partial^{i} \partial_{j} \frac{1}{\partial^{2}} \mathcal{F}^{j 0}=-\partial_{i}\left[-\partial_{0}^{2}+\nabla^{2}\right] \frac{1}{\partial^{2}} \mathcal{J}_{\Phi}=\mathcal{F}^{0 i} \quad, \quad F_{(1)}^{i j}=0 .
\end{aligned}
$$

Assuming a static and transverse vector potential yields,

$$
A_{(0)}^{\mu}(\eta, \vec{x})=(0, \vec{m} \times \vec{\nabla} \mathcal{A}(\vec{x})) \quad \Longrightarrow \quad \mathcal{F}^{0 i}=0 \quad, \quad \mathcal{F}^{i j}=\epsilon^{i j k}\left[m_{k} \nabla^{2}-\vec{m} \cdot \vec{\nabla} \partial_{k}\right] \mathcal{J}_{\mathcal{A}}
$$

where we define,

$$
\mathcal{J}_{\mathcal{A}}(x) \equiv-\int d^{4} x^{\prime} i\left[F_{(1)}\left(x ; x^{\prime}\right)+G_{(1)}\left(x ; x^{\prime}\right)\right] \mathcal{A}\left(\vec{x}^{\prime}\right) .
$$

Substituting (25) into (22) allows us to express the one loop field strengths in terms of $\nabla^{2} / \partial^{2} \mathcal{J}_{\mathcal{A}}$,

$$
\begin{aligned}
A_{(0)}^{\mu}(\eta, \vec{x}) & =(0, \vec{m} \times \vec{\nabla} \mathcal{A}(\vec{x})) \\
& \Longrightarrow F_{(1)}^{0 i}=-\epsilon^{i j k} m_{j} \partial_{k} \partial_{0}\left(\frac{\nabla^{2}}{\partial^{2}} \mathcal{J}_{\mathcal{A}}\right), \quad F_{(1)}^{i j}=\epsilon^{i j k}\left[m_{k} \nabla^{2}-\vec{m} \cdot \vec{\nabla} \partial_{k}\right]\left(\frac{\nabla^{2}}{\partial^{2}} \mathcal{J}_{\mathcal{A}}\right) .
\end{aligned}
$$

\section{The one-loop correction to the field strengths}

The purpose of this section is to derive the one loop field strengths for a point charge (sub-section IV A) and for a point magnetic dipole (sub-section IVB). In each case the computation is first made with the source at the origin in flat, conformal coordinates (see Fig. 2) and the observer at fixed $x \equiv\|\vec{x}\|$, which gives the result for an observer who is being pulled apart from the source by the expansion of the universe. We then transform to the frame of an observer at fixed physical distance from the source, according to the formulae given in Appendix A.

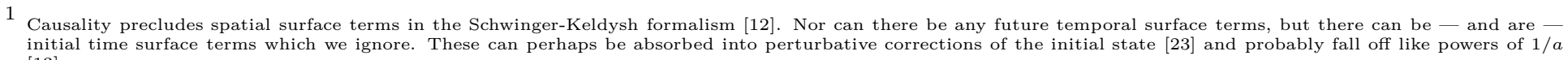
[13]. 


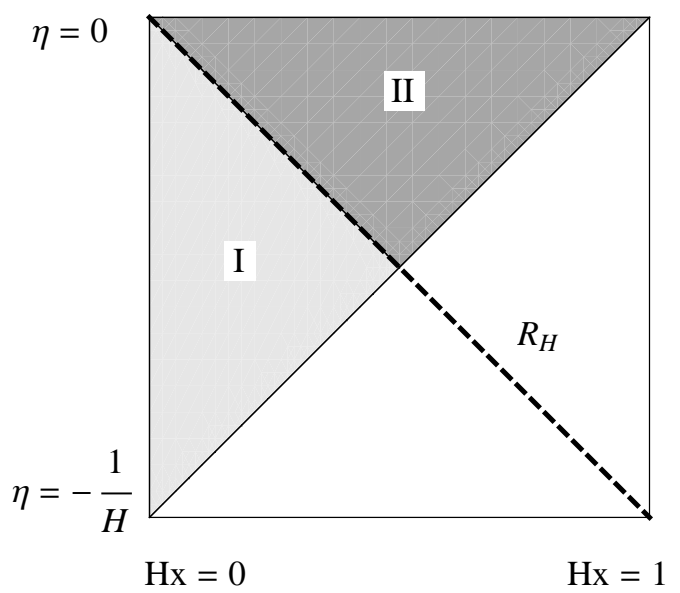

FIG. 2. The conformal diagram of de Sitter space. The universe is released at $\eta_{0}=-1 / H$ and we show only the region $\eta_{0}<\eta<0$ and $0 \leq x \leq 1 / H$. Our point sources are at $x=0$. Regions I and II are in causal contact with the initial instant of the sources. The dashed line at $x=-\eta$, corresponds to the physical distance (at fixed $\eta$ ) from the source being one Hubble radius $R_{H}=1 / H$. This line splits the causal region into Part I (sub-Hubble) and Part II (super-Hubble).

\section{A. Point charge}

The classical current of a static, point charge $q$ is

$$
J^{0}(\eta, \vec{x})=q \delta^{3}(\vec{x}), \quad J^{i}(\eta, \vec{x})=0
$$

Solving (18) gives the corresponding classical field strengths,

$$
E_{(0)}^{i} \equiv F_{(0)}^{i 0}(\eta, \vec{x})=-\partial_{i}\left(\frac{q}{4 \pi x}\right), \quad F_{(0)}^{i j}(\eta, \vec{x})=0 \quad(x=\|\vec{x}\|)
$$

Of course this corresponds to a static scalar potential of the type just considered. Substituting $\Phi_{(0)}=q / 4 \pi x$ in relations (23-24) allows us to express the nonzero one loop field strength in terms of derivatives of integrals $\mathcal{I}_{a b}$ which are given in Appendix B,

$$
\begin{aligned}
\kappa^{2} F_{(1)}^{0 i}(x)= & \frac{q}{4 \pi} \partial_{i}\left\{\frac{\kappa^{2} H^{2}}{8 \pi^{2} x}[\ln (a)+\alpha]-\frac{\kappa^{2}}{384 \pi^{3} a} \partial^{6}\left(\mathcal{I}_{22}-\mathcal{I}_{21}\right)\right. \\
& \left.+\frac{\kappa^{2} H^{2}}{32 \pi^{3}}\left[\left(\frac{1}{4} \partial^{4}+\partial_{0}^{2} \partial^{2}\right) \mathcal{I}_{12}+\left(-\frac{1}{4} \partial^{4}+\partial_{0}^{2} \partial^{2}\right) \mathcal{I}_{11}\right]\right\}
\end{aligned}
$$

Their derivatives are evaluated in Appendix C to produce the result,

$$
\begin{aligned}
\kappa^{2} F_{(1)}^{0 i}(\eta, \vec{x})= & \frac{q}{4 \pi} \partial_{i}\left\{\theta\left(\Delta \eta_{0}-x\right) \frac{\kappa^{2} H^{2}}{8 \pi^{2} x}\left[\frac{1}{3 a^{2} H^{2} x^{2}}+\ln (a H x)+\alpha-2 \ln \left(\frac{1-a^{-1}+H x}{1-a^{-1}-H x}\right)\right]\right. \\
& \left.+\theta\left(x-\Delta \eta_{0}\right) \frac{\kappa^{2} H^{2}}{8 \pi^{2} x}\left[\ln (a)+\alpha-4-\frac{1}{3(a-1)}-\frac{a}{3(a-1)^{2}}-3 \ln \left(1-\frac{1}{a}\right)\right]\right\}
\end{aligned}
$$

where $\Delta \eta_{0}=\eta-\eta_{0}=\eta+1 / H$ and $x=\|\vec{x}\|$. 
If we think of the full field strength as the curl of a covariant 4 -vector potential $A_{\mu} \equiv(\Phi, \vec{A})$, then expression (31) can be viewed as a one loop correction to the Coulomb potential,

$$
\begin{aligned}
\Phi_{(1)}=\Phi_{(0)} \times \frac{\kappa^{2} H^{2}}{8 \pi^{2}}\left\{\theta\left(\Delta \eta_{0}-x\right)\left[\frac{1}{3 a^{2} H^{2} x^{2}}+\ln (a H x)+\alpha-2 \ln \left(\frac{1-a^{-1}+H x}{1-a^{-1}-H x}\right)\right]\right. \\
\left.+\theta\left(x-\Delta \eta_{0}\right)\left[\ln (a)+\alpha-4-\frac{1}{3(a-1)}-\frac{a}{3(a-1)^{2}}-3 \ln \left(1-\frac{1}{a}\right)\right]\right\} .
\end{aligned}
$$

A number of points about this result deserve comment. First, note that nothing special happens at the Hubble radius, $x=-\eta$, which is the dashed line in Fig. 2. Second, it would be a mistake to pay much attention to the branch of (32) with $x>\Delta \eta_{0}$. As one can see from Fig. 2, observers in this region are not in causal contact with the point source; they only feel its influence as a consequence of whatever assumption is made about the long range fields which are present in the initial state. Understanding of this issue is in its infancy [23]. The need for perturbative corrections to the initial state is obvious from the constraint equations, and from the singularities which occur at $\eta=\eta_{0}$ on the last line of (32), but no one has worked out these corrections. The same comments apply to the logarithmic singularity on the first line of (32) which propagates along the light-cone from the initial appearance of the point source at $x^{\mu}=\left(\eta_{0}, \overrightarrow{0}\right)$. Note that all of these terms fall off at late time like powers of $1 / a$, which marks them as artifacts of the initial state.

The factor of $\alpha$ on the first line of (32) is also unimportant. It depends on whatever assumption we make about the finite parts of the counterterms (6-7). No physical principle can fix these counterterms in a nonrenormalizable theory such as Einstein + Maxwell [20] because they cannot be present on the fundamental level. They represent our ignorance about the ultraviolet completion of gravity and their appearance is one of the inevitable limitations of effective field theory [15, 21].

The reliable and significant parts of expression (32) are the factors of $1 / 3(a H x)^{2}$ and $\ln (a H x)$. The first of these is just the de Sitter descendant of the short distance enhancement that was found for flat space background [14, 15]. Its presence represents a nice correspondence check. The new, de Sitter feature is the enhancement factor of $\ln (a H x)$. Both features are plotted in Fig. 3,

When viewed at fixed $x$, the distinctively de Sitter factor of $\ln (a H x)$ in (32) grows linearly in the comoving time $\ln (a)=H t$. Because this secular enhancement factor multiplies the classical potential $\Phi_{(0)}$ it seems reasonable to regard the effect as a time-dependent renormalization of the source charge $q$, which contradicts the claim of Kitamoto and Kitazawa that infrared gravitons screen gauge couplings [10]. The slope is quite small. If one assumes single-scalar inflation, the measured value of the scalar power spectrum and the current limit on the tensor-to-scalar ratio [24] imply $\kappa^{2} H^{2} / 8 \pi^{2} \leq 3.3 \times 10^{-11}$. Nevertheless, the enhancement might be significant over a prolonged period of inflation. Of course one is limited by the reliability of perturbation theory; we cannot necessarily conclude that the effective charge grows past the 


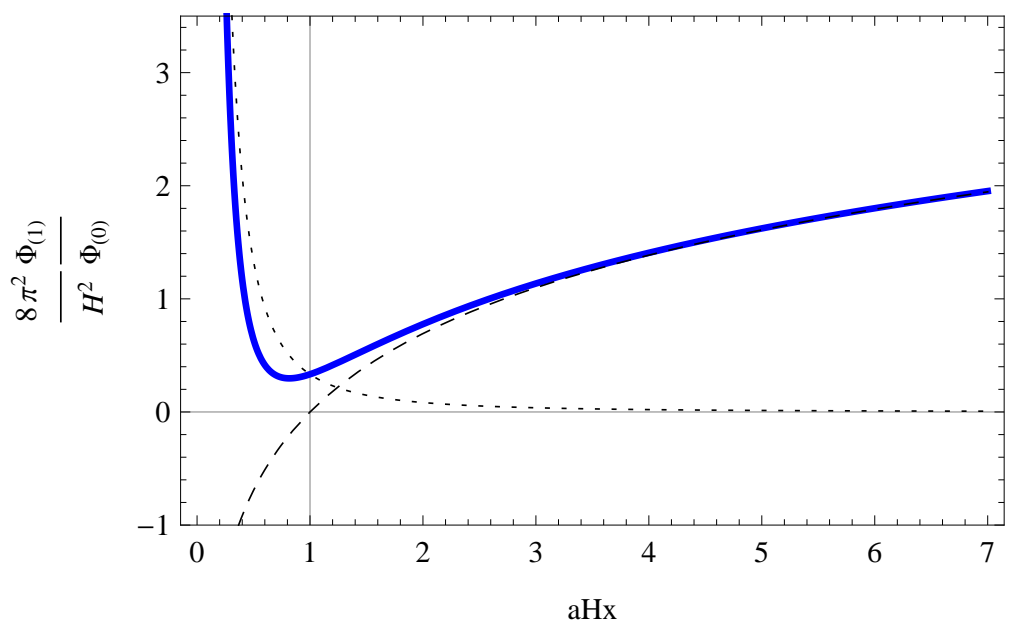

FIG. 3. The physically significant part of the ratio $\Phi_{(1)} / \Phi_{(0)}$ in units of $\kappa^{2} H^{2} / 8 \pi^{2}$, as a function of the physical distance in Hubble units, $a H x$. The solid blue curve gives $1 / 3 a^{2} H^{2} a^{2}+\ln (a H x)$. At short distances the ratio is dominated by $1 / 3 a^{2} H^{2} x^{2}$ (the short-dashed line). At large physical distances the ratio is dominated by $\ln (a H x)$ (the long-dashed line).

time at which $\kappa^{2} H^{2} / 8 \pi^{2} \times H t \sim 1$ because the higher loop contributions reach the same strength at this time. The reliable conclusion is rather that perturbation theory breaks down; one must employ some kind of nonperturbative resummation scheme to work out what happens later, for example [25].

The secular enhancement cannot be understood in the same terms as the screening from scalar quantum electrodynamics [3, 6] because both photon and graviton lines in the diagram of Fig. 1 are uncharged. The explanation seems to derive rather from the interpretation of force fields as transferring momentum by the exchange of virtual particles. The typical inflationary graviton carries a physical momentum of about $H$. There is little effect near the source because the virtual photons in this region carry much larger momenta. However, beyond a physical Hubble distance the momenta of inflationary gravitons is larger so scattering with them can give a virtual photon significantly more momentum than it would otherwise carry.

To understand how an isotropic ensemble of gravitons can still provide a net outward-directed push, consider the process in one spatial dimension. Suppose we add a random momentum $\Delta p$ to some fixed momentum $p>0$,

$$
p^{\prime}=p+\Delta p
$$

Even if the distribution of $\Delta p$ is symmetric about the origin the distribution of the magnitude of $p^{\prime}$ will still be asymmetric about $\left|p^{\prime}\right|=p$ because $\left|p^{\prime}\right|>p$ receives contributions from both $\Delta p>0$ and $\Delta p<-2 p$. In the latter case the scattered virtual photon has $p^{\prime}<-p$, so its momentum is delivered to the direction opposite from which it originally set out, but the force is still directed outward and stronger than without the scattering. The $\ln (a)=H t$ growth is the same "drunkard's walk" factor as the magnitude of a massless, minimally coupled scalar [26], which also receives stochastic accretions as successive modes experience horizon crossing.

These considerations are supported by the fields perceived by a an observer who is held at a fixed physical 
distance from the source. In Appendix A we transform the field strength (31) to the frame of this observer. Expressing the result (45) in terms of a one loop correction to the scalar potential gives,

$$
\widetilde{\Phi}_{(1)}=\widetilde{\Phi}_{(0)} \times \frac{\kappa^{2} H^{2}}{8 \pi^{2}}\left\{\frac{1}{3 H^{2} r^{2}}+\ln (H r)+\alpha-2 \ln \left(\frac{a-1+H r}{a-1-H r}\right)\right\},
$$

As before, only the first two terms are reliable and significant 2 However, both of these terms are constant, and the flat space factor $1 / 3 H^{2} r^{2}$ dominates the de Sitter correction $\ln (H r)$ for $H r<1$. That is just what one would expect because the typical inflationary gravitons responsible for the secular growth of (32) have Hubble-scale physical momenta. Only if one continues (34) to large values of $\mathrm{Hr}$ is the logarithmic enhancement apparent.

\section{B. Point magnetic dipole}

The current representing a point magnetic dipole of a strength $\vec{m}$ is

$$
J^{0}(\eta, \vec{x})=0, \quad J^{i}(\eta, \vec{x})=-\epsilon^{i j k} m_{j} \partial_{k} \delta^{3}(\vec{x}) .
$$

The classical field strength tensor associated with it is,

$$
F_{(0)}^{0 i}(\eta, \vec{x})=0, \quad F_{(0)}^{i j}(\eta, \vec{x})=\epsilon^{i j k}\left(m_{k} \nabla^{2}-\vec{m} \cdot \vec{\nabla} \partial_{k}\right) \frac{1}{4 \pi x} .
$$

Of course this system is described by a static and transverse vector potential of the form (25)), with $\mathcal{A}(\vec{x})=$ $1 / 4 \pi x$. Recall that the one loop field strengths for this case are based on the intermediate quantity $\mathcal{J}_{\mathcal{A}}(x)$ defined in expression (26). We can express it in terms of derivatives acting on the integrals $\mathcal{I}_{a b}$ given in Appendix B,

$$
\begin{aligned}
\kappa^{2} \mathcal{J}_{\mathcal{A}}(\eta, x) \equiv & -\int d^{4} x^{\prime} i\left[F_{(1)}\left(x ; x^{\prime}\right)+G_{(1)}\left(x ; x^{\prime}\right)\right] \frac{\kappa^{2}}{4 \pi\left\|\vec{x}^{\prime}\right\|}=-\frac{\kappa^{2} H^{2}}{8 \pi^{2}}\left[\frac{1}{3} \ln (a)-\alpha-\gamma\right] \frac{1}{4 \pi x} \\
& -\frac{a^{-1} \kappa^{2} \partial^{6}}{1536 \pi^{4}}\left(\mathcal{I}_{22}-\mathcal{I}_{21}\right)-\frac{\kappa^{2} H^{2}}{128 \pi^{4}}\left[\left(\frac{1}{12} \partial^{4}-\partial_{0}^{2} \partial^{2}\right) \mathcal{I}_{12}-\left(\frac{1}{12} \partial^{4}+\partial_{0}^{2} \partial^{2}\right) \mathcal{I}_{11}\right] .
\end{aligned}
$$

The various derivatives are acted in Appendix C to produce the result,

$$
\begin{aligned}
\kappa^{2} \mathcal{J}_{\mathcal{A}}(\eta, x) & =\frac{\kappa^{2} H^{2}}{8 \pi^{2}}\left\{\frac{\theta\left(\Delta \eta_{0}-x\right)}{4 \pi x}\left[\frac{1}{3 a^{2} H^{2} x^{2}}-\frac{1}{3} \ln (a H x)+\alpha+\gamma-2 \ln \left(\frac{1-a^{-1}+H x}{1-a^{-1}-H x}\right)\right]\right. \\
& \left.+\frac{\theta\left(x-\Delta \eta_{0}\right)}{4 \pi x}\left[-\frac{1}{3} \ln (a)+\alpha+\gamma-4-\frac{13}{3} \ln \left(1-\frac{1}{a}\right)-\frac{1}{3(a-1)}-\frac{a}{3(a-1)^{2}}\right]\right\},
\end{aligned}
$$

\footnotetext{
2 The last term in 34 is negligible when $a \gg 1$ (since it gets suppressed as $1 / a$ ) and the third term can be removed by a suitable choice of the counterterm.
} 
where $x=\|\vec{x}\|$ and $\Delta \eta_{0}=\eta+1 / H$.

All the comments we made after equation (32) apply as well to (38). In particular, the branch with $x>\Delta \eta_{0}$, which is not causally related to the point source on the initial value surface, is nonsense based on our having failed to perturbatively correct the initial state. Most of those terms also fall off like powers of $1 / a$. Even in the causal branch with $x<\Delta \eta_{0}$, the out-going and in-coming spherical wave is another artifact of the initial state, while the factors of $\alpha$ and $\gamma$ derive from the finite parts of higher derivative counterterms which parameterize our ignorance about the true ultraviolet completion of Einstein + Maxwell. As before, the terms which can be reliably fixed by low energy effective field theory are just the factors of $1 / 3 a^{2} H^{2} x^{2}$ and $-\frac{1}{3} \ln (a H x)$ on the first line of (효 $)$.

In the interests of simplicity we have used only the causal branch to compute the quantity $\nabla^{2} / \partial^{2} \mathcal{J}_{\mathcal{A}}$ which determines the one loop field strengths through relation (27),

$$
\begin{aligned}
& \frac{\nabla^{2}}{\partial^{2}} \kappa^{2} \mathcal{J}_{\mathcal{A}}=\frac{1}{4 \pi x} \times \frac{\kappa^{2} H^{2}}{8 \pi^{2}}\left\{\frac{1}{3 a^{2} H^{2} x^{2}}-\frac{2}{3} \ln (H x)+\alpha+\gamma-\frac{1}{3} \ln \left(\frac{a H x}{1+a H x}\right)\right. \\
& \left.\quad+\frac{5}{8} \ln \left(\frac{1-a^{-1}-H x}{1-a^{-1}+H x}\right)+\frac{1}{12} \ln \left(\frac{1+a^{-1}-H x}{1+a^{-1}+H x}\right)+\frac{\left(-\frac{3}{2}+\frac{2}{a}\right) H x}{\left(1-\frac{1}{a}\right)^{2}-H^{2} x^{2}}+\frac{\frac{1}{3}\left(1-\frac{1}{a}\right) H x}{\left[\left(1-\frac{1}{a}\right)^{2}-H^{2} x^{2}\right]^{2}}\right\} .
\end{aligned}
$$

Including the acausal branch would not affect the factors of $1 / 3 a^{2} H^{2} x^{2}$ and $-\frac{2}{3} \ln (H x)$ which are the only reliable and significant parts of (39). From expression (27) we see that the classical plus quantum vector potential is,

$$
\vec{A}(\eta, \vec{x})=\vec{m} \times \vec{\nabla}\left\{\frac{1}{4 \pi x}\left[1+\frac{\kappa^{2} H^{2}}{8 \pi^{2}}\left(\frac{1}{3 a^{2} H^{2} x^{2}}-\frac{2}{3} \ln (H x)+\text { Irrelevant }\right)+O\left(\kappa^{4}\right)\right]\right\} .
$$

Of course the factor of $1 / 3 a^{2} H^{2} x^{2}$ had to appear in (40) to give the correct flat space limit [14]. The striking things about the intrinsically de Sitter correction $-\frac{2}{3} \ln (H x)$, relative to the analogous correction to the point charge potential (32), are the opposite sign and the absence of secular growth at fixed $x$. These features mean that an observer at fixed $x$ perceives no secular change in the strength of the dipole, but different fixed $x$ observers report a screening of the dipole at increasing distance. It might be significant that the one loop corrections to the fields of a magnetic dipole from scalar quantum electrodynamics also show weaker time dependence than the corrections to the fields of a point charge [6], although the change in that case was from exponential screening to only linear screening.

We can use the formulae of Appendix A to transform the total (classical plus quantum) field strengths to the frame of an observer at a fixed physical distance $r=a x$ from the source. We shall only include the one 
loop terms from the factors of $1 / 3 a^{2} H^{2} x^{2}$ and $-\frac{2}{3} \ln (H x)$ in (40),

$$
\begin{aligned}
& \widetilde{F}_{0 i}(\tau, \vec{r})=e^{H \tau} \sqrt{1-H^{2} r^{2}} \frac{H \epsilon^{i j k} m^{j} \widehat{r}^{k}}{4 \pi r^{2}}\{1 \\
& \left.\quad+\frac{\kappa^{2} H^{2}}{8 \pi^{2}}\left[\frac{5}{H^{2} r^{2}}+\frac{2}{3}\left[2-\ln \left(\frac{H r}{a}\right)\right]+\text { Irrelevant }\right]+O\left(\kappa^{4}\right)\right\}, \\
& \widetilde{F}_{i j}(\tau, \vec{r})=\frac{e^{H \tau}}{\sqrt{1-H^{2} r^{2}}} \frac{\epsilon^{i j k}}{4 \pi r^{3}}\left\{m^{k}-3 \vec{m} \cdot \widehat{r} r^{k}\left[1-\frac{2}{3} H^{2} r^{2}\right]+\frac{\kappa^{2} H^{2}}{8 \pi^{2}}\left[\frac{3 m^{k}-5 \vec{m} \cdot \widehat{r} r^{k}}{H^{2} r^{2}}\right.\right. \\
& \left.\left.-\frac{2}{3} m^{k}\left[1+\ln \left(\frac{H r}{a}\right)\right]+2 \vec{m} \cdot \widehat{r} \widetilde{r}^{k}\left[\frac{2}{3}+\frac{2}{3} H^{2} r^{2}+\left(1-\frac{2}{3} H^{2} r^{2}\right) \ln \left(\frac{H r}{a}\right)\right]+\operatorname{Irrel} .\right]+O\left(\kappa^{4}\right)\right\} .
\end{aligned}
$$

(We remind the reader that the scale factor is $a=e^{H \tau} \sqrt{1-H^{2} r^{2}}$ in static coordinates.) Much of the complication in expression (42) is to make the magnetic field transverse in static coordinates, however, one can see that the classical fields experience a secular enhancement by the factor $\kappa^{2} H^{2} / 8 \pi^{2} \times \frac{2}{3} H \tau$. Of course this secular growth at fixed $r=a x$ is just the static coordinate reflection of the $\ln (H x)$ screening we found in the conformal coordinate result (40).

\section{Conclusion}

In this work we have studied how inflationary gravitons influence the electromagnetic field strengths of a point charge and a point magnetic dipole in de Sitter space. This was done by solving the quantum-corrected Maxwell's equations (11) at one loop order, using the vacuum polarization recently calculated in Ref. [13]. Results were derived for two types of observers, one at a fixed position in co-moving coordinates - and hence being pulled away from the source by the inflationary expansion — and one at a fixed physical distance from the source.

For a point charge the co-moving observer perceives a Coulomb potential (32) which seems to describe a secular renormalization of the charge by the factor $\kappa^{2} H^{2} / 8 \pi^{2} \times H t$. Even though the loop counting parameter is very small (the most recent data [24] implies $\kappa^{2} H^{2} / 8 \pi^{2} \leq 3.3 \times 10^{-11}$ if one assumes single-scalar inflation) this effect might be significant for a very long period of inflation. It also represents another entry in the growing list of secular effects mediated by inflationary gravitons [7, 11, 13].

The potential (34) of our static observer manifests only a logarithmic running of the charge in space by the factor $\kappa^{2} H^{2} / 8 \pi^{2} \times \ln (H r)$. The physical interpretation of both effects seems to be the momentum added to the force-carrying virtual photons by the ensemble of Hubble-scale gravitons ripped out of the vacuum by inflation. If these results and this interpretation stand up they would contradict the claim by Kitamoto and Kitazawa that inflationary gravitons screen gauge coupling constants [10]. 
The effect of inflationary gravitons on magnetic sources is weaker, just as was found in a recent study of the vacuum polarization from charged inflationary scalars [6]. Our co-moving observer perceives a vector potential (40) which contains no secular change in the dipole, although it is consistent with a logarithmic screening in space by the factor of $\kappa^{2} H^{2} / 8 \pi^{2} \times-\frac{2}{3} \ln (H x)$. Of course the static observer at fixed $r=a x$ perceives fields (41,42) which manifest a secular enhancement of the classical results by the factor $\kappa^{2} H^{2} / 8 \pi^{2} \times \frac{2}{3} H \tau$.

We should also comment on the gauge issue. The vacuum polarization from charged matter fields is gauge independent at one loop because it involves only matter field propagators. However, Fig. 1 shows that the contribution from gravitons involves potential gauge dependence from both the photon propagator and from the graviton propagator. An explicit study of this was made in flat space background, using the 3-parameter family of Poincaré invariant gauges [14]. Although the single photon gauge parameter dropped out there was massive dependence upon the two graviton gauge parameters. In fact the flat space structure function takes the form of a universal function whose form is dictated by dimensionality and Poincaré invariance, times an algebraic function of the graviton gauge parameters which can take any value on the real line [14]!

Of course this same gauge dependence must be present in the de Sitter vacuum polarization [13], otherwise it would not possess the correct flat space limit. However, the most important corrections are intrinsically de Sitter; that is, they carry factors of $H^{2}$ which vanish in the flat space limit. It has been suggested that factors of $H^{2} \ln (a)$ might be independent of the gauge [27]. They do have a clear physical origin in the continual production of inflationary gravitons. And there is precedence for this idea from the behavior of flat space Green's functions. These are highly gauge dependent, but they can be combined to give the gauge independent S-matrix. Fortunately, we are not reduced to opining about the possibility of gauge independence: the technology exists to check it [28, 29] and we have begun work on the project.

\section{Acknowledgements}

We are grateful to S. Deser and K. E. Leonard for conversations on this subject. This work is part of the D-ITP consortium, a program of the Netherlands Organisation for Scientific Research (NWO) that is funded by the Dutch Ministry of Education, Culture and Science (OCW), it was partially supported by NWO Veni Project \# 680-47-406 by NSF grant PHY-1205591, and by the Institute for Fundamental Theory at the University of Florida. 


\section{Appendix A - Static coordinates on de Sitter space}

The transformation from conformal coordinates to static coordinates $\widetilde{x}^{\mu}=(\tau, \vec{r})$ is,

$$
\tau=-\frac{1}{H} \ln \left[\frac{1}{a(\eta)} \sqrt{1-H^{2} r^{2}}\right], \quad r^{i}=a(\eta) x^{i},
$$

where $a(\eta)=-1 / H \eta$. The ranges are $-\infty<\tau<\infty, 0 \leq r=\|\vec{r}\|<1 / H$, and the invariant line element in these coordinates is,

$$
d s^{2}=-\left(1-H^{2} r^{2}\right) d \tau^{2}+\frac{d r^{2}}{1-H^{2} r^{2}}+r^{2} d \Omega^{2},
$$

where $d \Omega^{2}$ is the line element squared on the unit 2-sphere. In these coordinates an observer at $r$ is at a constant physical distance from the origin, where our point sources are placed.

The electromagnetic field strength tensor in static coordinates is given in terms of one in conformal coordinates as [] $]$,

$$
\begin{aligned}
& \widetilde{F}_{0 i}=\frac{e^{-2 H \tau}}{1-H^{2} r^{2}}\left[-F^{0 i}-H F^{i j} r^{j}\right], \\
& \widetilde{F}_{i j}=\frac{e^{-2 H \tau}}{\left(1-H^{2} r^{2}\right)^{2}}\left[\left(1-H^{2} r^{2}\right) F^{i j}-2 H^{2} r^{k} F^{k[i} r^{j]}-2 H F^{0[i} r^{j]}\right],
\end{aligned}
$$

where indices enclosed in square brackets are anti-symmetrized. Upon inserting (31) into Eq. (45)), one obtains (for a late time observer within the Hubble distance, $H r<\min [1, a-1]$ )

$$
\widetilde{F}_{0 i}(\vec{r})=-\frac{q}{4 \pi} \tilde{\partial}_{i}\left\{\frac{1}{r}+\frac{\kappa^{2} H^{2}}{8 \pi^{2} r}\left[\frac{1}{H^{2} r^{2}}+\ln (H r)+\alpha-2 \ln \left(\frac{a-1+H r}{a-1-H r}\right)\right]\right\}+\mathcal{O}\left(\kappa^{4}\right),
$$

where (covariant components of) the classical electric field is, $\widetilde{F}_{0 i}=-\tilde{\partial}_{i}[q /(4 \pi r)]=q r^{i} /\left(4 \pi r^{3}\right)$.

\section{Appendix B - Integrals from section IV}

The four integrals appearing in section IV are

$$
\mathcal{I}_{a b}=\int_{-1 / H}^{\eta} d \eta^{\prime} \int d^{3} x^{\prime} \frac{1}{\left\|\vec{x}^{\prime}\right\|} \theta\left(\eta-\eta^{\prime}-\left\|\vec{x}-\vec{x}^{\prime}\right\|\right) f_{a}\left(\eta^{\prime}, \vec{x}^{\prime}\right) g_{b}\left(\eta^{\prime}, \vec{x}^{\prime}\right), \quad(a, b=1,2),
$$

where

$$
f_{1}=1, \quad f_{2}=-H \eta^{\prime}, \quad g_{1}=1, \quad g_{2}=\ln \left[\frac{H^{2}}{4}\left(\left(\eta-\eta^{\prime}\right)^{2}-\left\|\vec{x}-\vec{x}^{\prime}\right\|^{2}\right)\right] .
$$

All the integrals are elementary, and they turn out to be

$$
\begin{aligned}
& \mathcal{I}_{11}=\theta\left(\Delta \eta_{0}-x\right) 4 \pi\left[\frac{x^{3}}{12}-\frac{x^{2} \Delta \eta_{0}}{6}+\frac{\Delta \eta_{0}^{3}}{6}\right]+\theta\left(x-\Delta \eta_{0}\right) 4 \pi \frac{\Delta \eta_{0}^{4}}{12 x} \\
& \mathcal{I}_{21}=-H \eta \mathcal{I}_{11}+\theta\left(\Delta \eta_{0}-x\right) 4 \pi H\left[\frac{\Delta \eta_{0}^{4}}{8}-\frac{\Delta \eta_{0}^{2} x^{2}}{12}+\frac{x^{4}}{40}\right]+\theta\left(x-\Delta \eta_{0}\right) 4 \pi H \frac{\Delta \eta_{0}^{4}\left[4 \Delta \eta_{0}-5 \eta\right]}{60 x},
\end{aligned}
$$




$$
\begin{aligned}
\mathcal{I}_{12}= & \theta\left(\Delta \eta_{0}-x\right) 4 \pi\left\{-\frac{1}{12 x}\left(\Delta \eta_{0}^{2}-x^{2}\right)\left(\Delta \eta_{0}-x\right)^{2} \ln \left[\frac{H}{2}\left(\Delta \eta_{0}-x\right)\right]\right. \\
& \left.+\frac{1}{12 x}\left(\Delta \eta_{0}^{2}-x^{2}\right)\left(\Delta \eta_{0}+x\right)^{2} \ln \left[\frac{H}{2}\left(\Delta \eta_{0}+x\right)\right]+\frac{x^{3}}{6} \ln (H x)-\frac{19}{72} x^{3}-\frac{4}{9} \Delta \eta_{0}\left(\Delta \eta_{0}^{2}-x^{2}\right)\right\} \\
& +\theta\left(x-\Delta \eta_{0}\right) 4 \pi\left\{\frac{\Delta \eta_{0}^{4}}{6 x} \ln \left(H \Delta \eta_{0}\right)-\frac{19}{72} \frac{\Delta \eta_{0}^{4}}{x}\right\}, \\
\mathcal{I}_{22}= & -H \eta \mathcal{I}_{12}+\theta\left(\Delta \eta_{0}-x\right) 4 \pi H\left\{\left[\frac{\Delta \eta_{0}^{5}}{15 x}+\frac{\Delta \eta_{0}^{4}}{8}-\frac{\Delta \eta_{0}^{2} x^{2}}{12}+\frac{x^{4}}{40}\right] \ln \left[\frac{H}{2}\left(\Delta \eta_{0}+x\right)\right]\right. \\
& \left.+\left[-\frac{\Delta \eta_{0}^{5}}{15 x}+\frac{\Delta \eta_{0}^{4}}{8}-\frac{\Delta \eta_{0}^{2} x^{2}}{12}+\frac{x^{4}}{40}\right] \ln \left[\frac{H}{2}\left(\Delta \eta_{0}-x\right)\right]-\frac{77 \Delta \eta_{0}^{4}}{240}+\frac{59}{360} \Delta \eta_{0}^{2} x^{2}-\frac{19}{400} x^{4}\right\} \\
& +\theta\left(x-\Delta \eta_{0}\right) 4 \pi\left\{\frac{2}{15} \frac{\Delta \eta_{0}^{5}}{x} \ln \left(H \Delta \eta_{0}\right)-\frac{46}{225} \frac{\Delta \eta_{0}^{5}}{x}\right\},
\end{aligned}
$$

where $x=\|\vec{x}\|$ and $\Delta \eta_{0}=\eta+1 / H$.

\section{Appendix C - Derivatives}

It is often necessary to act the flat space d'Alembertian on a function of the conformal $\eta$ and just the magnitude $x \equiv\|\vec{x}\|$ of the position vector. For this case we can write,

$$
\partial^{2} f(\eta, x)=\frac{1}{x}\left(\partial_{x}-\partial_{0}\right)\left(\partial_{x}+\partial_{0}\right)[x f(\eta, x)]
$$

This form is particularly effective when acting on out-going or in-coming spherical waves: $f(x-\eta) / x$ or $f(x+\eta) / x$.

The rest is straightforward but tedious. The various derivatives of section IV are,

$$
\begin{aligned}
\partial^{4} \mathcal{I}_{11} & =\frac{8 \pi}{x} \\
\partial_{0}^{2} \partial^{2} \mathcal{I}_{11} & =-\theta\left(x-\Delta \eta_{0}\right) \frac{8 \pi}{x} \\
\partial^{4} \mathcal{I}_{12} & =\theta\left(\Delta \eta_{0}-x\right) \frac{8 \pi}{x}[2 \ln (H x)+1]+\theta\left(x-\Delta \eta_{0}\right) \frac{8 \pi}{x}\left[2 \ln \left(1-\frac{1}{a}\right)+1\right] \\
\partial_{0}^{2} \partial^{2} \mathcal{I}_{12} & =\theta\left(\Delta \eta_{0}-x\right) \frac{8 \pi}{x} \ln \left[\frac{1-a^{-1}-H x}{1-a^{-1}+H x}\right]-\theta\left(x-\Delta \eta_{0}\right) \frac{8 \pi}{x}\left[2 \ln \left(1-\frac{1}{a}\right)+1\right] \\
\partial^{6} \mathcal{I}_{21} & =0 \\
\partial^{6} \mathcal{I}_{22} & =-\theta\left(\Delta \eta_{0}-x\right) \frac{16 \pi}{a x^{3}}+\theta\left(x-\Delta \eta_{0}\right) \frac{16 \pi H^{2}}{x}\left[\frac{a^{2}}{(a-1)^{2}}+\frac{a}{a-1}\right]
\end{aligned}
$$


Note that no delta functions appear from taking derivatives of step functions in (150 53 since the coefficients multiplying them are zero.

[1] A. A. Starobinsky, JETP Lett. 30 (1979) 682; V. F. Mukhanov and G. V. Chibisov, JETP Lett. 33 (1981) 532.

[2] T. Brunier, V. K. Onemli and R. P. Woodard, Class. Quant. Grav. 22 (2005) 59, gr-qc/0408080; E. O. Kahya and V. K. Onemli, Phys. Rev. D76 (2007) 043512, gr-qc/0612026.

[3] T. Prokopec, O. Törnkvist and R. P. Woodard, Phys. Rev. Lett. 89 (2002) 101301, astro-ph/0205331; Annals Phys. 303 (2003) 251, gr-qc/0205130; T. Prokopec and R. P. Woodard, Am. J. Phys. 72 (2004) 62, astro-ph/0303358; Annals Phys. 312 (2004) 1, gr-qc/0310056; T. Prokopec and E. Puchwein, JCAP 0404 (2004) 007, astro-ph/0312274.

[4] T. Prokopec and R. P. Woodard, JHEP 0310 (2003) 059, astro-ph/0309593; B. Garbrecht and T. Prokopec, Phys. Rev. D73 (2006) 064036, gr-qc/0602011; L. D. Duffy and R. P. Woodard, Phys. Rev. D72 (2005) 024023, hep-ph/0505156.

[5] S. Park and R. P. Woodard, Phys. Rev. D83 (2011) 084049, arXiv:1101.5804; Phys. Rev. D84 (2011) 124058, arXiv:1109.4187.

[6] H. Degueldre and R. P. Woodard, Eur. Phys. J. C73 (2013) 2457, arXiv:1303.3042.

[7] S. P. Miao and R. P. Woodard, Class. Quant. Grav. 23 (2006) 1721, gr-qc/0511140; Phys. Rev. D74 (2006) 024021, gr-qc/0603135 Class. Quant. Grav. 25 (2008) 145009, arXiv:0803.2377.

[8] S. P. Miao, arXiv:0705.0767; Phys. Rev. D86 (2012) 104051, arXiv:1207.5241,

[9] E. O. Kahya and R. P. Woodard, Phys. Rev. D76 (2007) 124005, arXiv:0709.0536; Phys. Rev. D77 (2008) 084012, arXiv:0710.5282,

[10] H. Kitamoto and Y. Kitazawa, Phys. Rev. D87 (2013) 124007, arXiv:1203.0391; Phys. Rev. D87 (2013) 124004, arXiv:1204.2876.

[11] P. J. Mora, N. C. Tsamis and R. P. Woodard, JCAP 1310 (2013) 018, arXiv:1307.1422.

[12] J. Schwinger, J. Math. Phys. 2 (1961) 407; K. T. Mahanthappa, Phys. Rev. 126 (1962) 329; P. M. Bakshi and K. T. Mahanthappa, J. Math. Phys. 4 (1963) 1; J. Math. Phys. 4 (1963) 12; L. V. Keldysh, Sov. Phys. JETP 20 (1965) 1018; K. C. Chou, Z. B. Su, B. L. Hao and L. Yu, Phys. Rept. 118 (1985) 1; R. D. Jordan, Phys. Rev. D33 (1986) 444; E. Calzetta and B. L. Hu, Phys. Rev. D35 (1987) 495.

[13] K. E. Leonard and R. P. Woodard, Class. Quant. Grav. 31 (2014) 015010, arXiv:1304.7265. 
[14] K. E. Leonard and R. P. Woodard, Phys. Rev. D85 (2012) 104048, arXiv:1202.5800.

[15] N. E. J. Bjerrum-Bohr, Phys. Rev. D66 (2002) 084023, hep-th/0206236.

[16] K. E. Leonard, T. Prokopec and R. P. Woodard, Phys. Rev. D87 (2013) 044030, arXiv:1210.6968.

[17] N. C. Tsamis and R. P. Woodard, Commun. Math. Phys. 162 (1994) 217; R. P. Woodard, gr-qc/0408002

[18] G. Kleppe, Phys. Lett. B317 (1993) 305.

[19] K. E. Leonard, T. Prokopec and R. P. Woodard, J. Math. Phys. 54 (2013) 032301, arXiv:1211.1342.

[20] S. Deser and P. van Nieuwenhuizen, Phys. Rev. Lett. 32 (1974) 245; Phys. Rev. D10 (1974) 401.

[21] J. F. Donoghue, Phys. Rev. Lett. 72 (1994) 2996, gr-qc/9310024; Phys. Rev. D50 (1994) 3874, gr-qc/9405057.

[22] L. H. Ford and R. P. Woodard, Class. Quant. Grav. 22 (2005) 1637, gr-qc/0411003.

[23] E. O. Kahya, V. K. Onemli and R. P. Woodard, Phys. Rev. D81 (2010) 023508, arXiv:0904.4811.

[24] Z. Hou et al., Astrophys. J. 782 (2014) 74, arXiv:1212.6267.

[25] T. Prokopec, N. C. Tsamis and R. P. Woodard, Annals Phys. 323 (2008) 1324, arXiv:0707.0847; Class. Quant. Grav. 24 (2007) 201, gr-qc/0607094; Phys. Rev. D78 (2008) 043523, arXiv:0802.3673.

[26] A. Vilenkin and L. H. Ford, Phys. Rev. D26 (1982) 1231; A. D. Linde, Phys. Lett. 116B (1982) 335; A. A. Starobinsky, Phys. Lett. 117B (1982) 175.

[27] S. P. Miao and R. P. Woodard, JCAP 1207 (2012) 008, arXiv:1204.1784,

[28] N. C. Tsamis and R. P. Woodard, J. Math. Phys. 48 (2007) 052306, gr-qc/0608069.

[29] P. J. Mora, N. C. Tsamis and R. P. Woodard, J. Math. Phys. 53 (2012) 122502, arXiv:1205.4468 